\title{
The Rogers-Ramanujan Identities
}

\author{
Fazlee Hossain $^{1}$, Sabuj Das ${ }^{2}$, Haradhan Kumar Mohajan ${ }^{3, *}$ \\ ${ }^{1}$ Department of Mathematics, University of Chittagong, Bangladesh \\ ${ }^{2}$ Department of Mathematics, Raozan University College, Bangladesh \\ ${ }^{3}$ Premier University, Chittagong, Bangladesh \\ *Corresponding author: haradhan1971@gmail.com
}

Received February 12, 2015; Revised March 28, 2015; Accepted April 01, 2015

\begin{abstract}
In 1894, Rogers found the two identities for the first time. In 1913, Ramanujan found the two identities later and then the two identities are known as The Rogers-Ramanujan Identities. In 1982, Baxter used the two identities in solving the Hard Hexagon Model in Statistical Mechanics. In 1829 Jacobi proved his triple product identity; it is used in proving The Rogers-Ramanujan Identities. In 1921, Ramanujan used Jacobi’s triple product identity in proving his famous partition congruences. This paper shows how to generate the generating function for $C^{\prime}(n), C_{1}^{\prime}(n), C^{\prime \prime}(n)$ and $C_{1}^{\prime \prime}(n)$, and shows how to prove the Corollaries 1 and 2 with the help of Jacobi's triple product identity. This paper shows how to prove the Remark 3 with the help of various auxiliary functions and shows how to prove The Rogers-Ramanujan Identities with help of Ramanujan's device of the introduction of a second parameter a.
\end{abstract}

Keywords: at most, auxiliary function, convenient, expansion, minimal difference, operator, Ramanujan's device

Cite This Article: Fazlee Hossain, Sabuj Das, and Haradhan Kumar Mohajan, "The Rogers-Ramanujan Identities." Turkish Journal of Analysis and Number Theory, vol. 3, no. 2 (2015): 37-42. doi: 10.12691/tjant-3-2-1.

\section{Introduction}

In this article, we give some related definitions of $P(n), C^{\prime}(n), P_{m}\left(n-m^{2}\right), C_{1}^{\prime}(n), C^{\prime \prime}(n)$, $P_{m}(n-m(m+1))$ and $C_{1}^{\prime \prime}(n)$. We describe the generating functions for $C^{\prime}(n), P_{m}\left(n-m^{2}\right), C_{1}^{\prime}(n)$, $C^{\prime \prime}(n), P_{m}(n-m(m+1))$ and $C_{1}^{\prime \prime}(n)$, and establish the Remarks 1 and 2 with numerical examples and also prove the Corollaries 1 and 2 with the help of Jacobi's triple product identity [3]. We transfer the auxiliary function into another auxiliary function with the help of Ramanujan's device of the introduction of a second parameter $a$ [5],

i.e.,

$$
G_{k}(a, x)=\prod_{n=0}^{\infty}(-1)^{n} a^{2 n} x^{\frac{n(5 n+1)-2 k n}{2}}\left(1-a^{k} x^{2 k n}\right) C_{n}
$$

to

$$
G_{1}(x, x)=\sum_{m=0}^{\infty}\left(1-x^{5 m+1}\right)\left(1-x^{5 m+4}\right)\left(1-x^{5 m+5}\right),
$$

where $k=1$, and $a=x$, it is used in proving The RogersRamanujan Identity 1 . We prove The Rogers-Ramanujan Identities with the help of auxiliary functions.

\section{Some Related Definitions}

$P(n)$ [7]: The number of partitions of $n$ like: $4,3+1$, $2+2,2+1+1,1+1+1+1 \therefore P(4)=5$.

$C^{\prime}(n)$ [6]: The number of partitions of $n$ into parts each of which is of one of the forms $5 m+1$ and $5 m+4$.

$P_{m}\left(n-m^{2}\right)$ : The number of partitions of $n-m^{2}$ into $m$ parts at most.

$C^{\prime \prime}(n)$ : The number of partitions of $n$ into parts of the forms $5 m+2$ and $5 m+3$.

$C_{1}^{\prime}(n)$ : The number of partitions of $n$ into parts without repetitions or parts whose minimal difference is 2 .

$P_{m}(n-m(m+1))$ : The number of partitions of $n-m(m+1)$ into $m$ parts at most.

$C_{1}^{\prime \prime}(n)$ : The number of partitions of $n$ into parts not less than 2 and with minimal difference 2 .

\section{Generating Functions for $C^{\prime}(n)$ and $C^{\prime \prime}(n)$}

In this section we describe the generating functions for $C^{\prime}(n)$ and $C^{\prime \prime}(n)$ respectively. The generating function for $C^{\prime}(n)$ is of the form [5]; 


$$
\begin{aligned}
& \sum_{m=0}^{\infty} \frac{1}{\left(1-x^{5 m+1}\right)\left(1-x^{5 m+4}\right)} \\
= & \frac{1}{(1-x)\left(1-x^{4}\right)\left(1-x^{6}\right)\left(1-x^{9}\right) \ldots \infty} \\
= & 1+x+x^{2}+x^{3}+2 x^{4}+2 x^{5}+3 x^{6}+\ldots \infty \\
= & 1+\sum_{n=1}^{\infty} C^{\prime}(n) x^{n}
\end{aligned}
$$

where the coefficient $C^{\prime}(n)$ of $x^{n}$ is the number of partitions of $n$ into parts each of which is of one of these forms $5 m+1$ and $5 m+4$.

Now we consider a special function, which is given below:

$$
\begin{aligned}
& \frac{x^{m^{2}}}{(1-x)\left(1-x^{2}\right) \ldots\left(1-x^{m}\right)} \\
= & x^{m^{2}} \sum_{n=m^{2}}^{\infty} P_{m}\left(n-m^{2}\right) x^{n-m^{2}} \\
= & \sum_{n=m^{2}}^{\infty} P_{m}\left(n-m^{2}\right) x^{n}
\end{aligned}
$$

It is convenient to define $P_{m}(0)=1$. The coefficient $P_{m}\left(n-m^{2}\right)$ of $x^{n}$ in the above expansion is the number of partitions of $n-m^{2}$ into $m$ parts at most. Another special function, which is defined as;

$$
\begin{aligned}
& +\sum_{m=1}^{\infty} \frac{x^{m^{2}}}{(1-x)\left(1-x^{2}\right) \ldots\left(1-x^{m}\right)} \\
= & 1+\frac{x}{1-x}+\frac{x^{4}}{(1-x)\left(1-x^{2}\right)} \\
& +\frac{x^{9}}{(1-x)\left(1-x^{2}\right)\left(1-x^{3}\right)}+\ldots \infty \\
= & 1+x+x^{2}+x^{3}+2 x^{4}+2 x^{5} \\
& +3 x^{6}+3 x^{7}+\ldots \infty \\
= & 1+\sum_{n=1}^{\infty} C_{1}^{\prime}(n) x^{n}
\end{aligned}
$$

where the coefficient $C_{1}^{\prime}(n)$ is the number of partitions of $n$ into parts without repetitions or parts, whose minimal difference is 2 .

From (1) and (2) we can establish the following Remark:

\section{Remark 1:}

$$
C_{1}^{\prime}(11)=C^{\prime}(11)
$$

i.e., the number of partitions of $n$ with minimal difference 2 is equal to the number of partitions of $n$ into parts of the forms $5 m+1$ and $5 m+4$.
Example 1: For $n=11$, there are 7 partitions of 11 that are enumerated by $C_{1}^{\prime}(n)$ of above statement, which are given bellow [6]:

$$
\begin{aligned}
& 11,10+1,9+2,8+3,7+4,7+3+1,6+4+1, \\
& \therefore C_{1}^{\prime}(11)=7 .
\end{aligned}
$$

There are 7 partitions of 11 are enumerated by $C_{1}^{\prime}(n)$ of above statement, which are given bellow:

$$
\begin{aligned}
& 11,9+1+1,6+4+1,6+1+1+1+1+1, \\
& 4+4+1+1+1,4+1+1+1+1+1+1+1, \\
& 1+1+1+1+1+1+1+1+1+1+1, \\
& \therefore C^{\prime}(11)=7 .
\end{aligned}
$$

Hence, $C_{1}^{\prime}(11)=C^{\prime}(11)$.

We can conclude that, $C_{1}^{\prime}(11)=C^{\prime}(11)$.

$$
\begin{aligned}
& 1+\sum_{n=1}^{\infty} C^{\prime}(n) x^{n}=1+\sum_{n=1}^{\infty} C_{1}^{\prime}(n) x^{n} . \\
& 1+\sum_{m=1}^{\infty} \frac{x^{m^{2}}}{(1-x)\left(1-x^{2}\right) \ldots\left(1-x^{m}\right)} \\
& =\sum_{m=0}^{\infty} \frac{1}{\left(1-x^{5 m+1}\right)\left(1-x^{5 m+4}\right)}
\end{aligned}
$$

which will be proved later as identity 1, it is known as The Rogers-Ramanujan identity 1.

The generating function for $C^{\prime \prime}(n)$ is of the form [1];

$$
\begin{aligned}
& \sum_{m=0}^{\infty} \frac{1}{\left(1-x^{5 m+2}\right)\left(1-x^{5 m+3}\right)} \\
= & \frac{1}{\left(1-x^{2}\right)\left(1-x^{3}\right)\left(1-x^{7}\right)\left(1-x^{8}\right) \ldots \infty} \\
= & 1+0 . x+x^{2}+x^{3}+x^{4}+x^{5}+2 x^{6}+2 x^{7}+\ldots \infty \\
= & 1+\sum_{n=1}^{\infty} C^{\prime \prime}(n) x^{n}
\end{aligned}
$$

where the coefficient $C^{\prime \prime}(n)$ is the number of partitions of $n$ into parts of the forms $5 m+2$ and $5 m+3$.

Now we consider a special function, which is of the form [1];

$$
\begin{aligned}
& \frac{x^{m(m+1)}}{(1-x)\left(1-x^{2}\right) \ldots\left(1-x^{m}\right)} \\
= & x^{m(m+1)} \sum_{n=m(m+1)}^{\infty} P_{m}(n-m(m+1)) x^{n-m(m+1)} \\
= & \sum_{n=}^{\infty} P_{m}(n-m(m+1)) x^{n},
\end{aligned}
$$

where the coefficient $P_{m}(n-m(m+1))$ of $x^{n}$ in the above expansion is the number of partitions of $n-m(m+1)$ into $m$ parts at most.

Another special function, which is defined as; 


$$
\begin{aligned}
& 1+\sum_{m=1}^{\infty} \frac{x^{m(m+1)}}{(1-x)\left(1-x^{2}\right) \ldots\left(1-x^{m}\right)} \\
= & 1+\frac{x^{2}}{1-x}+\frac{x^{6}}{(1-x)\left(1-x^{2}\right)} \\
& +\frac{x^{12}}{(1-x)\left(1-x^{2}\right)\left(1-x^{3}\right)}+\ldots \infty \\
= & 1+x^{2}+x^{3}+x^{4}+x^{5}+2 x^{6} \\
& +2 x^{7}+3 x^{8}+\ldots \infty \\
= & 1+\sum_{n=1}^{\infty} C_{1}^{\prime \prime}(n) x^{n},
\end{aligned}
$$

where the coefficient $C_{1}^{\prime \prime}(n)$ is the number of partitions of $n$ into parts not less than 2 and with minimal difference 2 .

From (4) and (5) we can establish the following Remark:

Remarks 2:

$$
C_{1}^{\prime \prime}(n)=C^{\prime \prime}(n),
$$

i.e., the number of partitions of $n$ into parts not less than 2 and with minimal difference 2 is equal to the number of partitions of $n$ into parts of the forms $5 m+2$ and $5 m+3$.

Example 2: If $n=11$, the four partitions of 11 into parts not less than 2 and with minimal difference 2 are given below:

$$
11,9+2,8+3,7+4 \text {. }
$$

Hence, $C_{1}^{\prime \prime}(11)=4$.

Again the four partitions of 11 into parts of the form $5 \mathrm{~m}$ +2 and $5 m+3$ are given as;

$$
8+3,7+2+2,3+3+3+2,3+2+2+2+2 \text {. }
$$

Hence, $C^{\prime \prime}(11)=4$.

$\therefore C_{1}^{\prime \prime}(11)=C^{\prime \prime}(11)$.

We can conclude that, $C_{1}^{\prime \prime}(n)=C^{\prime \prime}(n)$.

$$
\begin{aligned}
\text { i.e., } 1+\sum_{m=1}^{\infty} C_{1}^{\prime \prime}(n) x^{n}=1+\sum_{m=1}^{\infty} C^{\prime \prime}(n) x^{n} \\
1+\sum_{m=1}^{\infty} \frac{x^{m(m+1)}}{(1-x)\left(1-x^{2}\right) \ldots\left(1-x^{m}\right)} \\
=\prod_{m=0}^{\infty} \frac{1}{\left(1-x^{5 m+2}\right)\left(1-x^{5 m+3}\right)},
\end{aligned}
$$

which will be proved later as identity 2, it is known as The Rogers-Ramanujan identity 2.

Now we give two Corollaries, which are related to the Jacobi's triple product identity [3].

\section{Corollary 1:}

$$
\begin{aligned}
& \prod_{n=0}^{\infty}\left(1-x^{5 n+1}\right)\left(1-x^{5 n+4}\right)\left(1-x^{5 n+5}\right) \\
& =\sum_{n=-\infty}^{\infty}(-1)^{n} x^{\frac{n(5 n+3)}{2}} .
\end{aligned}
$$

Proof: From Jacobi’s Theorem [2] we have;

$$
\begin{aligned}
& \prod_{n=0}^{\infty}\left\{\left(1-x^{2 n}\right)\left(1+x^{2 n+1} z\right)\left(1+x^{2 n-1} z^{-1}\right)\right\} \\
& =\sum_{n=-\infty}^{\infty} x^{n^{2}} z^{n},
\end{aligned}
$$

for all $z$ except $z=0$, if $|x|<1$.

If we write $x^{5 / 2}$ for $x,-x^{3 / 2}$ for $z$ and replace $n$ by $n+$ 1 on the left hand side we obtain;

$$
\begin{aligned}
& \prod_{n=0}^{\infty}\left(1-x^{5 n+1}\right)\left(1-x^{5 n+4}\right)\left(1-x^{5 n+5}\right) \\
= & 1-x-x^{4}+x^{7}+x^{13}-\ldots \infty \\
= & \sum_{n=-\infty}^{\infty}(-1)^{n} x^{\frac{n(5 n+3)}{2}} .
\end{aligned}
$$

Hence, the Corollary.

\section{Corollary 2:}

$$
\begin{aligned}
& \prod_{n=0}^{\infty}\left(1-x^{5 n+2}\right)\left(1-x^{5 n+3}\right)\left(1-x^{5 n+5}\right) \\
= & \sum_{n=-\infty}^{\infty}(-1)^{n} x^{\frac{n(5 n+1)}{2}} .
\end{aligned}
$$

Proof: From Jacobi’s Theorem we have;

$$
\begin{aligned}
& \prod_{n=0}^{\infty}\left(1-x^{2 n}\right)\left(1+x^{2 n+1} z\right)\left(1+x^{2 n-1} z^{-1}\right) \\
= & \sum_{n=-\infty}^{\infty} x^{n^{2}} z^{n},
\end{aligned}
$$

for all $z$ except $z=0$, when $|x|<1$.

If we write $x^{5 / 2}$ for $x,-x^{1 / 2}$ for $z$ and replace $n$ by $n+$ 1 on the left hand side we obtain;

$$
\begin{aligned}
& \prod_{n=0}^{\infty}\left(1-x^{5 n+2}\right)\left(1-x^{5 n+3}\right)\left(1-x^{5 n+5}\right) \\
= & 1-x^{2}-x^{3}+x^{9}+x^{11}-\ldots \infty \\
= & \sum_{n=-\infty}^{\infty}(-1)^{n} x^{\frac{n(5 n+1)}{2}} .
\end{aligned}
$$

Hence the Corollary.

\section{The Rogers-Ramanujan Identities}

First we transfer the following auxiliary function into another auxiliary function. Let us consider the auxiliary function [1, 2] with $|x|<1$ and $|a|<1$.

$$
G_{k}(a, x)=\prod_{n=0}^{\infty}(-1)^{n} a^{2 n} x \frac{n(5 n+1)-2 k n}{2}\left(1-a^{k} x^{2 k n}\right) C_{n}(7)
$$

it is known as Ramanujan's device of the introduction of a second parameter $a$, where $k$ is 0,1 or 2 and $C_{0}=1$, 


$$
C_{n}=\frac{(1-a)(1-a x) \ldots\left(1-a x^{n-1}\right)}{(1-x)\left(1-x^{2}\right) \ldots\left(1-x^{n}\right)} .
$$

Hence,

$$
\begin{aligned}
G_{k}(a, x)=\sum_{n=0}^{\infty}\left[\begin{array}{c}
(-1)^{n} a^{2 n} x \frac{n(5 n+1)-2 k n}{2}\left(1-a^{k} x^{2 k n}\right) \\
(1-a)(1-a x) \ldots\left(1-a x^{n-1}\right) \\
\times \frac{(1-x)\left(1-x^{2}\right) \ldots\left(1-x^{n}\right)}{(1-a)(1-a x) \ldots \infty}
\end{array}\right] . \\
=\sum_{n=0}^{\infty}\left[\begin{array}{l}
(-1)^{n} a^{2 n} x \frac{n(5 n+1)-2 k n}{2} \\
\times \frac{1-a^{k} x^{2 k n}}{\left[(1-x)\left(1-x^{2}\right) \ldots\left(1-x^{n}\right)\right.} \\
{\left[\begin{array}{l}
\left(1-a x^{n}\right)\left(1-a x^{n+1}\right) \ldots \infty
\end{array}\right]}
\end{array}\right] \\
=\sum_{n=0}^{\infty}(-1)^{n} a^{2 n} x \frac{n(5 n+1)-2 k n}{2}\left(1-a^{k} x^{2 k n}\right) P_{n} Q_{n}(a)
\end{aligned}
$$

where $P_{n}=\prod_{r=1}^{n} \frac{1}{1-x^{r}}$,

$$
Q_{n}(a)=\prod_{r=n}^{\infty} \frac{1}{1-a x^{r}}=H_{k}(a, x)
$$

which is another auxiliary function, and it is used in proving The Rogers-Ramanujan Identities [1].

But from (7) we can easily verify that with $k=1,2$ and $a=x$.

$$
\begin{gathered}
G_{1}(x, x)=1-x-x^{4}+x^{7}+x^{13}-\ldots \infty \\
G_{1}(x, x)=\prod_{n=0}^{\infty}\left(1-x^{5 n+1}\right)\left(1-x^{5 n+4}\right)\left(1-x^{5 n+5}\right)
\end{gathered}
$$

(by Corollary 1 ).

$$
\begin{gathered}
G_{2}(x, x)=1-x^{2}-x^{3}+x^{9}+x^{11}-\ldots \infty \\
G_{2}(x, x)=\prod_{m=0}^{\infty}\left(1-x^{5 m+2}\right)\left(1-x^{5 m+3}\right)\left(1-x^{5 m+5}\right)_{(10)}
\end{gathered}
$$

(by Corollary 2).

From (8) we can also find that, if $k=1$ and $a=x$, then;

$$
\begin{aligned}
& H_{1}(x, x)=\frac{G_{1}(x, x)}{(1-x)\left(1-x^{2}\right)\left(1-x^{3}\right) \ldots \infty} \\
& =\frac{\prod_{m=0}^{\infty}\left(1-x^{5 m+1}\right)\left(1-x^{5 m+4}\right)\left(1-x^{5 m+5}\right)}{(1-x)\left(1-x^{2}\right)\left(1-x^{3}\right) \ldots \infty} \\
& =\prod_{m=0}^{\infty} \frac{1}{\left(1-x^{5 m+2}\right)\left(1-x^{5 m+3}\right)} .
\end{aligned}
$$

Again for $k=2$ and $a=x$, we get;

$$
\begin{aligned}
& H_{2}(x, x)=\frac{G_{2}(x, x)}{(1-x)\left(1-x^{2}\right)\left(1-x^{3}\right) \ldots \infty} \\
& =\frac{\prod_{m=0}^{\infty}\left(1-x^{5 m+2}\right)\left(1-x^{5 m+3}\right)\left(1-x^{5 m+5}\right)}{(1-x)\left(1-x^{2}\right)\left(1-x^{3}\right) \ldots \infty} \\
& =\prod_{m=0}^{\infty} \frac{1}{\left(1-x^{5 m+1}\right)\left(1-x^{5 m+4}\right)} .
\end{aligned}
$$

Now we can consider the following Remark [2].

Remark 3: $H_{k}-H_{k-1}=a^{k-1} \eta H_{3-k}$, where the operator $\eta$ is defined by $\eta f(a)=f(a x)$, and $k=1$ or 2 .

Proof: From (8) we have;

$$
\begin{aligned}
& H_{k}=H_{k}(a, x) \\
& =\sum_{n=0}^{\infty}(-1)^{n} a^{2 n} x \frac{n(5 n+1)-2 k n}{2}\left(1-a^{k} x^{2 k n}\right) P_{n} Q_{n}(a),
\end{aligned}
$$

where $P_{n}=\prod_{r=11-x^{r}}^{n}$, and $Q_{n}(a)=\prod_{r=n}^{\infty} \frac{1}{1-a x^{r}}$,

It is convenient to define $P_{0}=1, H_{0}=1$. We have;

$H_{k}-H_{k-1}$

$=\sum_{n=0}^{\infty}\left\{\begin{array}{l}(-1)^{n} a^{2 n} x^{\frac{n(5 n+1)}{2}} \\ \times\left[\begin{array}{l}x^{-k n}-a^{k} x^{k n}-x^{(1-k) n} \\ +a^{k-1} x^{n(k-1)}\end{array}\right] P_{n} Q_{n}\end{array}\right\}$

$=\sum_{n=0}^{\infty}(-1)^{n} a^{2 n} x^{\frac{n(5 n+1)}{2}} \times\left[\begin{array}{l}a^{k-1} x^{n(k-1)}\left(1-a x^{n}\right) \\ +x^{-k n}\left(1-x^{n}\right)\end{array}\right] P_{n} Q_{n}$.

Now we have, $\left(1-a x^{n}\right) Q_{n}=Q_{n+1} \quad$ and $\left(1-x^{n}\right) P_{n}=P_{n-1}$, hence,

$$
\begin{aligned}
& H_{k}-H_{k-1} \\
& =\sum_{n=0}^{\infty}(-1)^{n} a^{2 n+k-1} x \frac{n(5 n+1)+2 n(k-1)}{2} P_{n} Q_{n+1} \\
& +\sum_{n=0}^{\infty}(-1)^{n} a^{2 n} x \frac{n(5 n+1)-2 k n}{2} P_{n-1} Q_{n} .
\end{aligned}
$$

In the second sum on the right hand side of the Identity we change $n$ into $n+1$. Thus,

$$
\begin{aligned}
& H_{k}-H_{k-1} \\
& =\sum_{n=0}^{\infty}(-1)^{n} a^{2 n+k-1} x \frac{n(5 n+1)+2 n(k-1)}{2} P_{n} Q_{n+1} \\
& -\sum_{n=0}^{\infty}(-1)^{n} a^{2(n+1)} x \frac{(n+1)(5 n+6)-2 k(n+1)}{2} P_{n} Q_{n+1} .
\end{aligned}
$$




$$
\begin{aligned}
& =\sum_{n=0}^{\infty}(-1)^{n}\left\{\begin{array}{l}
a^{2 n+k-1} x \frac{n(5 n+1)+2 n(k-1)}{2} \\
-a^{2(n+1)} x \frac{(n+1)(5 n+6)-2 k(n+1)}{2}
\end{array}\right\} P_{n} Q_{n+1} \\
& =\sum_{n=0}^{\infty}(-1)^{n}\left\{\begin{array}{l}
a^{2 n+k-1} x^{\frac{n(5 n+1)+2 n(k-1)}{2}} \\
\otimes\left(1-a^{3-k} x^{(2 n+1)(3-k)}\right)
\end{array}\right\} P_{n} Q_{n+1} \\
& =\sum_{n=0}^{\infty}(-1)^{n}\left[a^{k-1} \eta\left\{\begin{array}{c}
a^{2 n} x \\
\times\left(1-a^{3-k} x^{2 n(3-k)}\right)
\end{array}\right\}\right] P_{n} Q_{n+1} .
\end{aligned}
$$

We have $Q_{n+1}=\eta Q_{n}$ and so,

$$
\begin{aligned}
& H_{k}-H_{k-1} \\
& =a^{k-1} \eta \sum_{n=0}^{\infty}(-1)^{n} a^{2 n} x \frac{n(5 n+1)-2 n(3-k)}{2}\left(1-a^{3-k} x^{2 n(3-k)}\right) P_{n} Q_{n} \\
& =a^{k-1} \eta H_{3-k} .
\end{aligned}
$$

Hence, the Remark.

The Rogers-Ramanujan Identities

\section{Identity 1 [4]:}

$$
\begin{aligned}
& 1+\sum_{m=1}^{\infty} \frac{x^{m^{2}}}{(1-x)\left(1-x^{2}\right) \ldots\left(1-x^{m}\right)} \\
& =\prod_{m=0}^{\infty} \frac{1}{\left(1-x^{5 m+2}\right)\left(1-x^{5 m+3}\right)} .
\end{aligned}
$$

\section{Identity 2 [4]:}

$$
\begin{aligned}
& 1+\sum_{m=1}^{\infty} \frac{x^{m(m+1)}}{(1-x)\left(1-x^{2}\right) \ldots\left(1-x^{m}\right)} \\
& =\prod_{m=0}^{\infty} \frac{1}{\left(1-x^{5 m+2}\right)\left(1-x^{5 m+3}\right)} .
\end{aligned}
$$

Proof: From (8) we have;

$$
H_{k}(a, x)=\frac{G_{k}(a, x)}{(1-a)(1-a x) \ldots \infty}
$$

where $H_{0}=0$.

From above Remark we have;

$$
H_{k}-H_{k-1}=a^{k-1} \eta H_{3-k}
$$

where the operator $\eta$ is defined by $\eta f(a)=f(a x)$, and $k=1$ or 2. In particular

$$
\begin{gathered}
H_{1}=\eta H_{2}, \\
H_{2}-H_{1}=a \eta H_{1} .
\end{gathered}
$$

So we have,

$$
H_{2}=\eta H_{2}+a \eta^{2} H_{2}
$$

Suppose now that;

$$
H_{2}=1+c_{1} a+c_{2} a^{2}+\ldots \infty .
$$

where the coefficients depend on $x$ only. Substituting this into (15), we obtain;

$$
\begin{aligned}
& 1+c_{1} a+c_{2} a^{2}+\ldots \infty \\
= & 1+c_{1} a x+c_{2} a^{2} x^{2}+\ldots \infty+a\left(1+c_{1} a x^{2}+c_{2} a^{2} x^{4}+\ldots \infty\right) .
\end{aligned}
$$

Hence, equating the coefficients of various powers of a from both sides we get;

$$
\begin{aligned}
& c_{1}=\frac{1}{1-x}, c_{2}=\frac{x^{2}}{1-x^{2}} c_{1}, c_{3}=\frac{x^{4}}{1-x^{3}} c_{2}, \cdots, \\
& c_{n}=\frac{x^{n(n-1)}}{(1-x)\left(1-x^{2}\right) \ldots\left(1-x^{n}\right)} .
\end{aligned}
$$

From (13) and (16), we have for $k=2$;

$$
\begin{aligned}
& \frac{G_{2}(a, x)}{(1-a)(1-a x) \ldots \infty} \\
& =H_{2}(a, x) \\
& =1+\frac{a}{1-x}+\frac{a^{2} x^{2}}{(1-x)\left(1-x^{2}\right)} \\
& +\frac{a^{3} x^{6}}{(1-x)\left(1-x^{2}\right)\left(1-x^{3}\right)}+\ldots \infty .
\end{aligned}
$$

If $a=x$, then;

$$
\begin{aligned}
& 1+\frac{x}{1-x}+\frac{x^{4}}{(1-x)\left(1-x^{2}\right)} \\
& +\frac{x^{9}}{(1-x)\left(1-x^{2}\right)\left(1-x^{3}\right)}+\ldots \infty \\
& =\frac{G_{2}(x, x)}{(1-x)\left(1-x^{2}\right) \ldots \infty} 。
\end{aligned}
$$

Therefore,

$$
\begin{aligned}
& 1+\sum_{m=1}^{\infty} \frac{x^{m^{2}}}{(1-x)\left(1-x^{2}\right) \ldots\left(1-x^{m}\right)} \\
& =\prod_{m=0}^{\infty} \frac{1}{\left(1-x^{5 m+1}\right)\left(1-x^{5 m+4}\right)}
\end{aligned}
$$

Hence the Identity 1.

Again from (13), (14) and (16) we have with $k=1$,

$$
\begin{aligned}
& \frac{G_{1}(a, x)}{(1-a)(1-a x) \ldots \infty} \\
& =H_{1}(a, x)=\eta H_{2}(a, x) \\
& =1+\frac{a x}{1-x}+\frac{a^{2} x^{4}}{(1-x)\left(1-x^{2}\right)} \\
& +\frac{a^{3} x^{9}}{(1-x)\left(1-x^{2}\right)\left(1-x^{3}\right)}+\ldots \infty .
\end{aligned}
$$

If $a=x$, then we have; 


$$
\begin{aligned}
& 1+\frac{x^{2}}{1-x}+\frac{x^{6}}{(1-x)\left(1-x^{2}\right)} \\
& +\frac{x^{12}}{(1-x)\left(1-x^{2}\right)\left(1-x^{3}\right)}+\ldots \infty \\
& =\frac{G_{1}(x, x)}{(1-x)\left(1-x^{3}\right) \ldots \infty} .
\end{aligned}
$$

Therefore,

$$
\begin{aligned}
& 1+\sum_{m=1}^{\infty} \frac{x^{m(m+1)}}{(1-x)\left(1-x^{2}\right) \ldots\left(1-x^{m}\right)} \\
& =\prod_{m=0}^{\infty} \frac{1}{\left(1-x^{5 m+2}\right)\left(1-x^{5 m+3}\right)} .
\end{aligned}
$$

Hence the Identity 2.

\section{Conclusion}

In this study, we have shown $C_{1}^{\prime}(n)=C^{\prime}(n)$ with the help of a numerical example when $n=11$, and also have shown $C_{1}^{\prime \prime}(n)=C^{\prime \prime}(n)$ with the help of a numerical example when $n=11$. We have transferred the auxiliary function into another auxiliary function with the help of Ramanujan's device of the introduction of a second parameter $a$, i.e.,

$$
G_{k}(a, x)=\prod_{n=0}^{\infty}(-1)^{n} a^{2 n} x \frac{n(5 n+1)-2 k n}{2}\left(1-a^{k} x^{2 k n}\right) C_{n}
$$

to

$$
G_{2}(x, x)=\sum_{m=0}^{\infty}\left(1-x^{5 m+2}\right)\left(1-x^{5 m+3}\right)\left(1-x^{5 m+5}\right),
$$

where $k=2$, and $a=x$, it is used in proving The RogersRamanujan Identity 2. Finally we have proved The RogerRamanujan Identities with the help of auxiliary function,

$$
H_{k}(a, x)=\frac{G_{k}(a, x)}{(1-a)(1-a x) \ldots \infty},
$$

where $H_{0}=0$.

\section{References}

[1] Andrews, G.E, “An Introduction to Ramanujan's Lost Notebook”, American Mathmatical Monthly, 86: 89-108. 1979.

[2] Hardy, G.H. and Wright, E.M. "Introduction to the Theory of Numbers", $4^{\text {th }}$ Edition, Oxford, Clarendon Press, 1965.

[3] Jacobi, C.G.J. (1829), "Fundamenta Nova Theoriae Functionum Ellipticarum (in Latin), Konigsberg Borntraeger”, Cambridge University Press, 2012.

[4] Baxter, R.J., "Exactly Solved Model in Statistical Models", London, Academic Press, 1982.

[5] Ramanujan, S., “Congruence Properties of Partitions”, Math, Z. 9: 147-153. 1921.

[6] Ramanujan, S., "Some Properties of $P(n)$, Number of Partitions of $n$ ”, Proc. of the Cam. Philo. Society XIX, 207-210. 1919.

[7] Das, S. and Mohajan, H.K., "Generating Function for P(n,p,*) and P(n, *,p)”, Amer. Rev. of Math. and Sta. 2(1): 33-35. 2014. 